\title{
Discernment of large semiprimes with unknown factors
}

\author{
Tejas R. Rao
}

September 22, 2018

\begin{abstract}
We apply methodology used by Pocklington to derive a criterion to prove very large numbers are semiprime without knowing their factors or being able to find the factors without trial division, which would often be too computationally complex. The algorithm converges in roughly the same time it takes to test for the primality of a Proth number of a similar size.
\end{abstract}

\section{Introduction}

In this paper, we adapt the methodology of Pocklington, Brillhart, Lehmer, and Selfridge (1). Let us begin with a proposition and an explanation of the subtleties of finding large semiprimes with unknown factors. Let $N=m p+1$, where $p$ is prime and $m$ is a positive integer.

Proposition 1. For any $a \in \mathbb{N}$, if $N^{1 / 3}<p<N^{1 / 2}$ and

$$
a^{N-1} \equiv 1 \bmod N
$$

but,

$$
\operatorname{gcd}\left(a^{\frac{N-1}{p}}-1, N\right)=1
$$

then $N$ is prime or semiprime.

If $\operatorname{gcd}\left(a^{\frac{N-1}{p}}-1, N\right)=1$, we know $a^{\frac{N-1}{p}} \not \equiv 1 \bmod n_{i}$ for any prime factor $n_{i}$ of $N$. But $a^{N-1} \equiv 1 \bmod N$, implies $a^{N-1} \equiv 1 \bmod n_{i}$. Therefore, $p \mid \operatorname{ord}_{n_{i}}(a)$. Since $n_{i}$ is prime, we can write

$$
p\left|\operatorname{ord}_{n_{i}}(a)\right| \phi\left(n_{i}\right)=n_{i}-1 .
$$

Therefore, $n_{i} \equiv 1 \bmod p$ and thus $n_{i} \geq p>N^{1 / 3}$. Also, $N / n_{1} n_{2} n \equiv 1 \bmod p$. But $N / n_{1} n_{2}<N^{1 / 3}<2 p+1$, so $N / n_{1} n_{2}$ is precisely 1 , and thus $N$ can have at most two proper prime factors. The condition $p<N^{1 / 2}$ is specified because otherwise $N$ can only be prime by the same method.

Many methods can be used to show $N$ is not prime, in which case its semiprimality has been proven. The problem with this proposition is that its factors can be easily found. Specifically, since we know $N$ has precisely two factors, we can write $N=(x p+1)(y p+1)=$ 
$p(x y p+x+y)+1=m p+1$. But since $x y p^{2}<N<p^{3}$, we know that $x y<p$. Therefore, $x+y<p$ and thus $m \bmod p=x+y$ and $m-m \bmod p=x y p$. With these two equations in a system, $x$ and $y$ and thus $x p+1$ and $y p+1$ may be found. To obscure these factors, we need to prove $N$ is semiprime in a more roundabout manner.

\section{Formula}

Let $N=m p+1$, where $p$ is prime and $m$ is a positive integer.

Theorem 1. For any $a \in \mathbb{N}$, if $N^{1 / 4}<p<N^{1 / 3}$ and

$$
a^{N-1} \equiv 1 \bmod N
$$

but,

$$
\operatorname{gcd}\left(a^{\frac{N-1}{p}}-1, N\right)=1
$$

then $N$ is prime, semiprime, or has precisely three prime factors.

If $\operatorname{gcd}\left(a^{\frac{N-1}{p}}-1, N\right)=1$, we know $a^{\frac{N-1}{p}} \not \equiv 1 \bmod n_{i}$ for any prime factor $n_{i}$ of $N$. But $a^{N-1} \equiv 1 \bmod N$, implies $a^{N-1} \equiv 1 \bmod n_{i}$. Therefore, $p \mid \operatorname{ord}_{n_{i}}(a)$. Since $n_{i}$ is prime, we can write

$$
p\left|\operatorname{ord}_{n_{i}}(a)\right| \phi\left(n_{i}\right)=n_{i}-1 .
$$

Therefore, $n_{i} \equiv 1 \bmod p$ and thus $n_{i} \geq p>N^{1 / 4}$. Also, $N / n_{1} n_{2} n_{3} \equiv 1 \bmod p$. But $N / n_{1} n_{2} n_{3}<N^{1 / 4}<2 p+1$, so $N / n_{1} n_{2} n_{3}$ is precisely 1 , and thus $N$ can have at most three proper prime factors. The condition $p<N^{1 / 3}$ is specified because otherwise $N$ can have at most two proper prime factors by the same method.

Various methods can be used to show $N$ is not prime. For example, since primes are Fermat probable primes for every coprime base $b$, a prime $N$ will satisfy

$$
b^{N-1} \equiv 1 \bmod N \text {. }
$$

If this does not occur for some $b, N$ is composite.

Theorem 2. If $N$ satisfies the conditions in Theorem 1, then $N$ has exactly three proper factors if and only if there is an entirely positive integer solution $(x, y, z)$ of

$$
\begin{aligned}
x+y+z & =m \bmod p \\
x y z & =\frac{m-m \bmod p^{2}}{p^{2}} ; \\
(x+y) z+x y & =\frac{m \bmod p^{2}-m \bmod p}{p} .
\end{aligned}
$$


For necessity, if $N$ is sufficiently large and has exactly three proper factors and it satisfies the conditions of the first theorem, then we can write

$$
\begin{aligned}
N & =(x p+1)(y p+1)(z p+1) \\
& =p\left(x y z p^{2}+((x+y) z+x y) p+x+y+z\right)+1 \\
& =m p+1 .
\end{aligned}
$$

But since $p>N^{1 / 4}$, we know that $p\left(x y z p^{2}\right)<N<p^{4}$, and thus $x y z<p$. But if $x y z<p$, then $x+y+z<p$ for integer values greater than one. This means that $m \bmod p=x+y+z$. Also, since $(x+y) z+x y+x+y+z<p^{2}$ for sufficiently large values, $m \bmod p^{2}-m$ $\bmod p=((x+y) z+x y) p+x+y+z-(x+y+z)$. Dividing by $p$, we get $((x+y) z+x y)$. Also because of this, $m-m \bmod p^{2}=x y z p^{2}$. Dividing by $p^{2}$, we arrive at the result.

For sufficiency, we know that we can write $N=(x p+1)(y p+1)(z p+1)$ by the converse of the above logic. But since the conditions from theorem one are satisfied, $N$ can have at most three proper factors and thus $(x p+1),(y p+1)$, and $(z p+1)$ are prime.

Remark 1. If $p>N^{1 / 3}$ and $N$ is semiprime, then $N=(x p+1)(y p+1)=p(x y p+x+y)+1$ can be factored. Since $p^{2} x y<N<p^{3}, x+y<x y<p$. But then $x+y=m \bmod p$ and $p x y=m-m \bmod p$. With these two equations, $(x, y)$ may be attained. To avoid this, we specify $N^{1 / 4}<p<N^{1 / 3}$. When $N^{1 / 4}<p<N^{1 / 3}$, we uniquely meet three conditions: there are at most three proper factors, the inequalities in the proof of Theorem 2 hold, and $x+y$ is often greater than $p$ (in fact always greater than $p$ for values nearer to $N^{1 / 4}$ ). The third condition means that $x+y \neq m \bmod p$ often. This means that finding factors would be too computationally complex as one would have to utilize trial division as one can only do to factor small numbers. By only restricting $N$ to having at most three proper factors with the size constraints on $p$ and taking the extra time to prove $N$ is not prime and does not have precisely three proper factors, we can obscure the factors of a semiprime $N$.

Remark 2. For $p$ composite, Theorem 1 will apply if for each prime factor $p_{i}$ of $p$,

$$
\operatorname{gcd}\left(a^{\frac{N-1}{p_{i}}}-1, N\right)=1 .
$$

Furthermore, Theorem 2 and Remark 1 apply as they do for a prime $p$.

\section{Implementation}

For sufficiently large $N$ (10 digits or more is a generous lower bound), the implementation is as follows. Setting the constants in Theorem 2 as follows,

$$
\begin{aligned}
x+y+z & =a ; \\
x y z & =b ; \\
(x+y) z+x y & =c,
\end{aligned}
$$


and solving the system of equations, we arrive at

$$
\begin{aligned}
x & =\frac{\sqrt[3]{2 a^{3}+3 \sqrt{3} \sqrt{4 a^{3} b-a^{2} c^{2}-18 a b c+27 b^{2}+4 c^{3}}-9 a c+27 b}}{3 \sqrt[3]{2}} \\
& -\frac{\sqrt[3]{2}\left(3 c-a^{2}\right)}{3 \sqrt[3]{2 a^{3}+3 \sqrt{3} \sqrt{4 a^{3} b-a^{2} c^{2}-18 a b c+27 b^{2}+4 c^{3}}-9 a c+27 b}}+\frac{a}{3} .
\end{aligned}
$$

Check if this is an integer; if not, $N$ is semiprime. If $x$ is an integer, then since $x y z$ and $x+y+z$ are both integers, $y$ and $z$ must also be integers. Therefore, if and only if $x$ is not an integer, $N$ is semiprime. This final condition is not at all computationally complex, as explained in step 4 below.

To prove $N$ is semiprime, we thus do the following. Choose $N=m p+1$, where $N^{1 / 4}<p<$ $N^{1 / 3}$. Show the following:

$$
\begin{aligned}
a^{N-1} & \equiv 1 \bmod N \\
1 & =g c d\left(a^{\frac{N-1}{p}}-1, N\right) \\
b^{N-1} & \not \equiv 1 \bmod N \\
x & =\frac{\sqrt[3]{2 a^{3}+3 \sqrt{3} \sqrt{4 a^{3} b-a^{2} c^{2}-18 a b c+27 b^{2}+4 c^{3}}-9 a c+27 b}}{3 \sqrt[3]{2}} \\
& -\frac{\sqrt[3]{2}\left(3 c-a^{2}\right)}{3 \sqrt[3]{2 a^{3}+3 \sqrt{3} \sqrt{4 a^{3} b-a^{2} c^{2}-18 a b c+27 b^{2}+4 c^{3}}-9 a c+27 b}}+\frac{a}{3}, \\
x & \notin \mathbb{N}
\end{aligned}
$$

Here is the method for proving the aforementioned conditions. For steps 1, 2, and 3, the BigInteger class is utilized, allowing for arbitrary precision integers. For step 4, the BigDecimal class is utilized, allowing for arbitrary precision decimals $(5,6)$. Each step must be completed to move on to the next.

\section{Step 1: Modular Powers.}

We calculate $a^{p} \bmod N$ and $a^{\frac{N-1}{p}} \bmod N$ using modular exponentiation and the process of repeated squaring. Utilizing multithreading, this can be done concurrently (7). Then, with one modular multiplication, we calculate $a^{N-1} \bmod N$ by multiplying the former two results. This makes the implementation of step 1 converge in roughly the time it takes to test the primality of a Proth number of a similar size $(2,3,4)$.

\section{Step 2: Check $N$ is Composite}

Using similar methodology to step 1 , we find a coprime $b$ such that

$$
b^{N-1} \not \equiv 1 \bmod N .
$$

\section{Step 3: Greatest Common Divisor.}


Then, to calculate $\operatorname{gcd}\left(a^{N-1}-1, N\right)$, we iterate the Euclidean algorithm (8). Namely,

$$
\operatorname{gcd}(\alpha, \beta)=\operatorname{gcd}(\beta, \alpha \bmod \beta) .
$$

Step 2 also converges in roughly the time it takes to test the primality of a Proth number of a similar size.

\section{Step 4: Testing Theorem 2}

Theorem 2 is far less computationally complex. Utilize the BigDecimal class and calculate the value of $x$ shown above. Since the largest monomials in the expression are about equal to $p^{3}<N$, the calculation of $(x, y, z)$ will be significantly shorter than $a^{N-1} \bmod N$, which requires $z$ modular powers of numbers of a similar size, where $2^{z}$ is the largest power of 2 dividing $N-1$. To solve for $x, y$, or $z$ in the implementation of Theorem 2 , the number of multiplications and roots is far below 100, which itself is far below $z$ for any significantly sized input $N$.

\section{References}

[1] Brillhart, John; Lehmer, D. H.; Selfridge, J. L.

New Primality Criterion and Factorizations of $2^{m} \pm 1$

Mathematics of Computation, (1975) 29 (130): 620-647

[2] Rao, T. R.

An open source software package for primality testing of numbers of the form $\mathrm{p} 2 \mathrm{n}+1$, with no constraints on the relative sizes of $\mathrm{p}$ and $2 \mathrm{n}$.

PeerJ, (2018)

[3] Rao, T. R.

Primitive Indexes, Zsigmondy Numbers, and Primoverization.

Cornell University Library, (2018)

[4] Rao, T. R.

Effective Primality Test for $p 2^{n}+1, p$ prime, $n>1$.

Cornell University Library, (2018)

[5] Oracle.

Class BigInteger.

Oracle, (n.d.)

[6] Oracle.

Class BigDecimal.

Oracle, (n.d.)

[7] Intel.

Intel Hyper-Threading Technology.

Intel, (2010) 
Tejas R. Rao

[8] Jones, G. A.; Jones, J. M.

Bezout's Identity.

Elementary Number Theory, (1998) 7-11 